\title{
An application of PCC model: risk measurement of extreme event in Chinese stock market
}

\author{
Bai Manying ${ }^{1, a}$, Ma Fanghui, ${ }^{2, b}$, Guo Fengjuan ${ }^{3, c}$ \\ ${ }^{1,2,3}$ School of Economics and Management, Beihang University, No. 37, Xueyuan Road, Haidian \\ District, Beijing 100191, China \\ aemail: baimy805@gmail.com
}

Keywords: pair-copula construction; exteme event loss; Monte Carlo

\begin{abstract}
We focus on analyzing extreme event on stock market with the pair-copula constructions (PCC) based multivariate models with GARCH (p, q) margins. We utilize the PCC model to get the estimation of joint PDF parameters. Then, we use six indices construct the decomposition of the PCC copula. As for different tail dependence of these log-returns series, we build the estimating model with bivariate t-copulas. Finally, we apply Monte Carlo method to simulate the extreme loss with parameters estimated from decomposing steps.
\end{abstract}

\section{Introduction}

Extreme event happens in finance market and it could generate huge loss to the investors even though its frequency is low. In this paper, we focus on the stock market violation and we are intending to observe the high dimensional factors' decomposition so as to analysis the extreme risk measure and management of the stock market based on the pair-copula theory. We use the paircopula constructions (PCC) to obtain the joint probability distribution based on multivariable data series. The appearance of pair-copula theory supplies a kind of way to solve the problem of decomposing the high dimensional copula. And Pair-copula well solved the problem of estimating the high dimensional copula parameters.

This paper intends to analysis of extreme event on Chinese stock market. We first introduce the relevant theory and methodology, and then give the empirical analysis. Section 2 gives the paircopula construction model. Section 3 uses six indices to construct the decomposition of the PCC copula and build the estimating model with bivariate t-copulas. Finally, we apply Monte Carlo method to simulate the extreme loss with parameters. Section 4 concludes this paper.

\section{Pair-copula Construction}

\subsection{Decomposition of pair-copula for multivariate density functions}

Consider a n-dimensional random variables $X=\left(X_{1}, X_{2}, \ldots X_{n}\right)$ with a joint density function $f\left(x_{1}, x_{2}, \ldots x_{n}\right)$. The density can be factorized uniquely as

$$
f\left(x_{1}, x_{2}, \ldots x_{n}\right)=f\left(x_{1}\right) \cdot f\left(x_{2} \mid x_{1}\right) \cdot f\left(x_{3} \mid x_{1}, x_{2}\right) \cdot \ldots f\left(x_{n} \mid x_{1}, \ldots x_{n-1}\right)
$$

We can get the pair-copula decomposition formula:

$$
f\left(x_{1}, x_{2}, \ldots x_{n}\right)=\prod_{r=1}^{n} f\left(x_{r}\right) \times \prod_{j=1}^{n-1} \prod_{i=1}^{n-j} c_{j, j+i \mid 1, \cdots, j-1}(j=t-k, j+i=t)
$$

In the next we need to know the exact decomposition step of the copula function $c_{j, j+i \mid 1, \cdots, j-1}$.

\section{Numerical analysis: an application of PCC to the stock market}

We utilize the above model to the stock market. We consider six time series of daily log-returns 
of six indices' prices as follows: industrial index (GY); public utility (GS); finance index (JR); Consumer Discretionary (KX); consumer staples index (RX); Information Technology Index (XJ). The data's resource is wind database, and the period is between December 31st, 2009 and December 31st, 2011.

\subsection{Estimation of marginal distribution}

We use log-return series of industrial index (GYR); public utility (GSR); finance index (JRR); Consumer Discretionary (KXR); consumer staples index (RXR); Information Technology Index (XJR), and we take the lowest daily price of each index as the sample of log-returns. Here we apply the GARCH $(1,1)-t$ model to the log-return series and obtain the standardized residuals to describe the marginal distribution of return series with conditional heteroscedasticity .

Table1 gives the parameter estimation of GARCH(1,1)-t for the six log-return series. We could see that each parameter pass the test on a high level of significance, and thus each log-return can be described by GARCH(1,1)-t. After the estimation of each parameter, we could get the daily logreturn distribution of the six indices.

Table 1. Parameter estimation of GARCH model

\begin{tabular}{cccccc}
\hline & Parameter 0 & Parameter & Parameter & Parameter & $\begin{array}{c}\text { Degree of } \\
\text { Free }\end{array}$ \\
\hline GY & 0.020 & 0.093 & 0.046 & 0.920 & 5.338 \\
(P Value) & $(0.017)$ & $(0.021)$ & $(0.029)$ & $(0.000)$ & $(0.000)$ \\
GS & 0.032 & 0.120 & 0.057 & 0.884 & 4.813 \\
(P Value) & $(0.028)$ & $(0.020)$ & $(0.033)$ & $(0.000)$ & $(0.000)$ \\
JR & -0.062 & 0.070 & 0.035 & 0.928 & 3.451 \\
(P Value) & $(0.019)$ & $(0.031)$ & $(0.018)$ & $(0.000)$ & $(0.000)$ \\
KX & -0.004 & 0.074 & 0.048 & 0.926 & 6.116 \\
(P Value) & $(0.010)$ & $(0.021)$ & $(0.032)$ & $(0.000)$ & $(0.002)$ \\
RX & 0.103 & 0.058 & 0.087 & 0.893 & 7.396 \\
(P Value) & $(0.001)$ & $(0.010)$ & $(0.005)$ & $(0.000)$ & $(0.027)$ \\
XJ & 0.008 & 0.118 & 0.071 & 0.897 & 8.856 \\
(P Value) & $(0.009)$ & $(0.020)$ & $(0.030)$ & $(0.000)$ & $(0.035)$ \\
\hline
\end{tabular}

\subsection{Pair-copula decomposition}

We use these standardized residuals generated from GARCH model as the data series so as to choose the pair-copula function. Figure 1 is the scatter plots of each pair of marginal distribution series. From the figure we could see obvious upper and lower tail dependence.

Table 2 gives the estimation results and the shadowed cells are the number we choose to get the root node. From the table we could see that node JR has the most shadowed cells, hence node JR is the root node we seek. 



Fig. 1. Scatter plots of the unit interval transformed standardized residuals

Table 2. Estimated degree of free in a bivariate t-copula model

\begin{tabular}{llllll}
\hline & GS & JR & KX & RX & XJ \\
\hline GY & 3.373 & 4.258 & 2.346 & 3.530 & 2.648 \\
GS & & 2.316 & 3.226 & 3.064 & 4.395 \\
JR & & & 2.257 & 1.825 & 2.458 \\
KX & & & & 3.905 & 4.581 \\
RX & & & & & 6.482 \\
\hline
\end{tabular}

Table 3. Estimated start and final ML parameters

\begin{tabular}{ccccc}
\hline Parameters & \multicolumn{2}{c}{ Start } & \multicolumn{2}{c}{ Final } \\
\hline & Correlation & Degree of free & Correlation & Degree of free \\
14 & 0.972 & 2.352 & 0.972 & 2.346 \\
23 & 0.800 & 2.320 & 0.800 & 2.316 \\
34 & 0.753 & 2.261 & 0.752 & 2.257 \\
35 & 0.561 & 1.825 & 0.560 & 1.825 \\
36 & 0.616 & 2.458 & 0.616 & 2.458 \\
$24 \mid 3$ & 0.803 & 5.343 & 0.796 & 5.372 \\
$25 \mid 3$ & 0.674 & 9.103 & 0.675 & 9.103 \\
$26 \mid 3$ & 0.790 & 8.643 & 0.795 & 8.640 \\
$13 \mid 4$ & -0.280 & 10.204 & -0.288 & 10.194 \\
$12 \mid 34$ & -0.271 & 16.988 & -0.269 & 17.324 \\
$45 \mid 23$ & 0.598 & 8.637 & 0.597 & 8.625 \\
$56 \mid 23$ & 0.582 & 16.324 & 0.581 & 16.323 \\
$15 \mid 234$ & 0.004 & 24.877 & 0.004 & 25.947 \\
$46 \mid 235$ & -0.333 & 27.761 & -0.332 & 27.760 \\
$16 \mid 2345$ & -0.319 & 6398385 & -0.318 & 6398370 \\
MLE & \multicolumn{2}{c}{1957.319} & & \multicolumn{2}{c}{1957.438} \\
\hline
\end{tabular}

Table 3 lists the result of pair-copula's parameters. We got the initial value and the final value during the whole estimation, and we would use the final value together with the parameters of marginal distribution to construct the joint probability distribution of the whole market.

\subsection{Monte Carlo simulation result}

Here we set change rate $7 \%$ as the trigger of extreme event and utilize Monte Carlo method to simulate the distribution. According to the result, the probability of downward change rate over $7 \%$ in the next day is less than $5 \%$, meanwhile, the probability of downward change rate over $8 \%$ is less than $1 \%$. And we could see these probabilities as the small probability event which means there is little chance of breaking out financial crisis or fierce violation of stock market. 


\section{Conclusion}

This paper presents an analysis of extreme event on stock market with the PCC copula based multivariate models with GARCH (p, q) margins.

The results show that, pair-copula could well describe the dependence structure of different risk factors and during the decomposing process, we could adjust the pair-copula function used in different tree so as to find the most appropriate structure, this is also the most important reason we choose pair-copula model to value the risk factors. PPC model has wider application beyond our article, such as insurance portfolio investment and futures market. In this context, we focus on the application of forecasting the probability of extreme event occurrence. PPC model provides us a more flexible way to measure the composite risk a market faces; moreover, it well presents the dependence structure of the risk factors, which is extremely practical because the composite index is too composite to observe the interaction of different factors.

\section{Acknowledgements}

The authors thank the National Science Foundation of China (71071009) for providing financial support for this work.

\section{References}

[1] D. Berg,K. Aas. Models for construction of multivariate dependence[J]. Norwegian Computing Center, Technical Report. 2007:

[2] K. Aas,C. Czado,A. Frigessiet al. Pair-copula constructions of multiple dependence[J]. Insurance: Mathematics and Economics. 2009. 44(2): 182-198

[3] C. Genest,H. U. Gerber,M. J. Goovaertset al. Editorial to the special issue on modeling and measurement of multivariate risk in insurance and finance[J]. Insurance: Mathematics and Economics. 2009. 44 (2) : 143-145

[4] Y. Liu,R. Luger. Efficient estimation of copula-GARCH models[J]. Computational Statistics \& Data Analysis. 2009. 53 (6) : 2284-2297

[5] Y. H. Lai,C. W. S. Chen. Optimal dynamic hedging via copula-threshold-GARCH models[J]. Mathematics and Computers in Simulation. 2009. 79 (8) : 2609-2624 